\title{
COVID-19 Pneumonia or Hypereosinophilic Syndrome?
}

\author{
Istemi Serina, d, Avni Ulusoy ${ }^{\mathrm{b}}$, Mediha Irem Onar ${ }^{\mathrm{b}}$, Mehmet Hilmi Dogu ${ }^{\mathrm{c}}$
}

\begin{abstract}
Hypereosinophilic syndromes (HESs) are a group of disorders characterized by pathological proliferation of eosinophils. Diagnostic criteria include eosinophil count of $1,500 / \mathrm{mm}^{3}$ or higher, presence of organ involvement and exclusion of other causes of eosinophilia for at least 6 months. A 69-year-old male patient was referred to the pandemic clinic with a preliminary diagnosis of coronavirus disease 2019 (COVID-19) with fever and dyspnea. Computed tomography (CT) was compatible with COVID-19, nasopharyngeal swab polymerase chain reaction (PCR) was negative for two times. He had $20,000 / \mathrm{mm}^{3}$ eosinophilia and retrospective examinations showed that he have had eosinophilia for more than 1 year. Platelet-derived growth factor receptor alpha $(P D G F R \alpha)$ resulted positively. After starting parenteral methylprednisolone with a dose of $1 \mathrm{mg} / \mathrm{kg}$, he was discharged with oral maintenance therapy. In outpatient follow-up, it was observed that eosinophilic pneumonia completely regressed. HES may occur with multiple system and organ involvement and findings. In the differential diagnosis of patients presenting with heart failure, pulmonary involvement and eosinophilia, HES must definitely be considered.
\end{abstract}

Keywords: COVID-19; Eosinophilia; Hypereosinophilic syndrome; Pulmonary involvement

\section{Introduction}

Hypereosinophilic syndromes (HESs) are a group of disorders characterized by excessive proliferation of eosinophils, with eosinophilic infiltration and mediator release. Although it has been considered idiopathic for a long time, etiologies have been described for some forms of HES. Percentage of eosinophils in peripheral blood should be $3-5 \%$; its absolute value

Manuscript submitted September 18, 2020, accepted September 26, 2020

Published online October 21, 2020

aDepartment of Hematology, University of Health Sciences, Istanbul Training and Research Hospital, Istanbul, Turkey

${ }^{b}$ Department of Internal Medicine, University of Health Sciences, Bagcilar Training and Research Hospital, Bagcilar, Istanbul, Turkey

'Deparment of Internal Medicine and Hematology, Liv Hospital ULUS, Istanbul, Turkey

${ }^{\mathrm{d} C o r r e s p o n d i n g ~ A u t h o r: ~ I s t e m i ~ S e r i n, ~ D e p a r t m e n t ~ o f ~ H e m a t o l o g y, ~ U n i v e r s i t y ~}$ of Health Sciences, Istanbul Training and Research Hospital, Org. Nafiz GURMAN Cad., 34098 Fatih, Istanbul, Turkey. Email: serinistemi@hotmail.com

doi: https://doi.org/10.14740/jmc3587 is between $350-500 / \mathrm{mm}^{3}$ [1]. Eosinophilia, which expresses the increase of eosinophils in peripheral blood, is examined by grading mild $\left(500-1,500 / \mathrm{mm}^{3}\right)$, moderate $(1,500-5,000 /$ $\left.\mathrm{mm}^{3}\right)$ and heavy $\left(>5,000 / \mathrm{mm}^{3}\right)[1,2]$. The term "hypereosinophilia" is generally used for values above $1,500 / \mu \mathrm{L}$ [3]. In the data of "The Surveillance, Epidemiology and End Results (SEER)" between 2001 and 2005, the incidence of HES was determined as 0.036 per 100,000 [4]. Fatigue, cough, shortness of breath, myalgia, angioedema, rhinitis, rash and fever are the most common symptoms of hypereosinophilia. The most common clinical findings in the follow-up of patients with hypereosinophilia are due to dermatological involvement $(69 \%)$. Then there are pulmonary $(44 \%)$ and gastrointestinal (38\%) findings [5]. Eosinophilia-induced cardiomyopathy has been reported at $20 \%$ [6]. Progressive heart failure is the most typical example of eosinophilic organ damage and develops as a result of a multi-step pathophysiological process.

During the coronavirus disease 2019 (COVID-19) pandemic, which affects the whole world, the patient was referred to emergency clinic with cough, high fever, and pneumonic infiltration was detected in lung imaging. In our case, a patient who was referred to the pandemic service with suspicion of COVID-19 based on clinical and imaging results, but was diagnosed with HES through an advanced examination, is mentioned.

\section{Case Report}

A 69-year-old male patient with New York Heart Association stage IV (NYHA IV) heart failure and chronic obstructive pulmonary disease (COPD) was admitted to our hospital's emergency clinic with fever, shortness of breath and cough. Polymerase chain reaction (PCR) was planned with thorax imaging and nasopharyngeal swab, considering COVID-19 in the foreground. In thorax computed tomography, patched ground glass consolidation areas were observed at the level of the middle and lower lobe segments of the lung (Fig. 1). In his examinations, hemoglobin was $12.3 \mathrm{~g} / \mathrm{dL}$, white blood cell count 56,410/ $\mathrm{mm}^{3}$, neutrophil was $31,720 / \mathrm{mm}^{3}$, eosinophil was $20,000 / \mathrm{mm}^{3}$, platelet was $402,000 / \mathrm{mm}^{3}$, urea was $34 \mathrm{mg} / \mathrm{dL}$, and creatinine $0.66 \mathrm{mg} / \mathrm{dL}$. Lactate dehydrogenase (LDH) was $312 \mathrm{mg} / \mathrm{dL}$ and C-reactive protein (CRP) was $35 \mathrm{mg} / \mathrm{dL}$. Retrospective examinations showed that he have had eosinophilia for more than 1 year. The peripheral smear evaluated for remarkable eosinophilia has increased white blood cell count and eosinophils, and toxic granulation is observed in neutrophils. In stool microscopy examined for secondary reasons, trophozoite, parasitic cyst and egg were not observed. Malignancy and additional pathology were not detected in the neck, thorax, and abdominal 


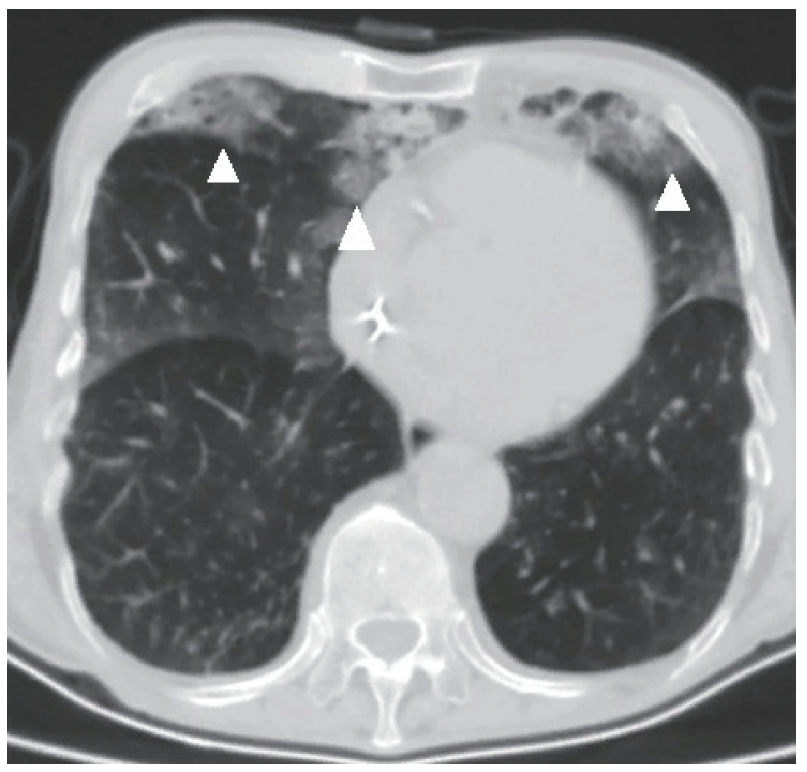

Figure 1. Pulmonary involvement of HES, computed tomography at initial diagnosis: patchy images of HES involvement (arrows). There was no pathological lymphadenomegaly in the mediastinum. In the evaluation of the lung parenchyma structures, ground glass patchy consolidation areas were observed at the level of the middle and lower lobe segments. Ground glass densities were determined at the level of the upper lobe segments, compatible with proliferative phase COVID-19 pneumonia. HES: Hypereosinophilic syndrome; COVID-19: coronavirus disease 2019.

computed tomography. The COVID-19 PCR sample was found to be negative twice. Platelet-derived growth factor receptor alpha $(P D G F R \alpha)$ mutation was found positive in genetic mutation screening. Bronchoalveolar lavage (BAL) was planned for pulmonary involvement of HES, but it could not be performed due to the risk of transmission in pandemic situation.

He was taking furosemide $80 \mathrm{mg} /$ day and digoxin tablet $0.5 \mathrm{mg} /$ day orally for congestive heart failure, and had also an implantable cardioverter defibrillator (ICD) for 2 years. Methylprednisolone with a dose of $1 \mathrm{mg} / \mathrm{kg}$ was added to the treatment of the patient who was evaluated as HES lung involvement. The hemogram after 5 days was as follows; hemoglobin: $9.5 \mathrm{~g} / \mathrm{dL}$, white blood cell: $7,430 / \mathrm{mm}^{3}$, neutrophil: $3,830 / \mathrm{mm}^{3}$, eosinophil: $180 / \mathrm{mm}^{3}$, platelet: $403,000 / \mathrm{mm}^{3}$. The patient was discharged with oral maintenance methylprednisolone treatment. In the outpatient follow-up, 3 weeks after beginning methylprednisolone, it was observed through computed tomography that the signs of eosinophilic pneumonia completely regressed (Fig. 2), and his complaints entirely disappeared. While the steroid dose was planned to be reduced, it was planned to continue with imatinib mesylate $100 \mathrm{mg}$ daily. Diagnostic criteria of HES include eosinophil count 1,500/ $\mathrm{mm}^{3}$ or higher for at least 6 months, presence of organ involvement signs (often heart, lung, central nervous system and peripheral nervous system) and other causes of eosinophilia (parasitic disease, allergic diseases, immune deficiencies and malignancy) are excluded. The main pathology of HEP is the sequestration of eosinophils in organ tissues or systems. Eosinophil derivative neurotoxin, eosinophil cationic protein and

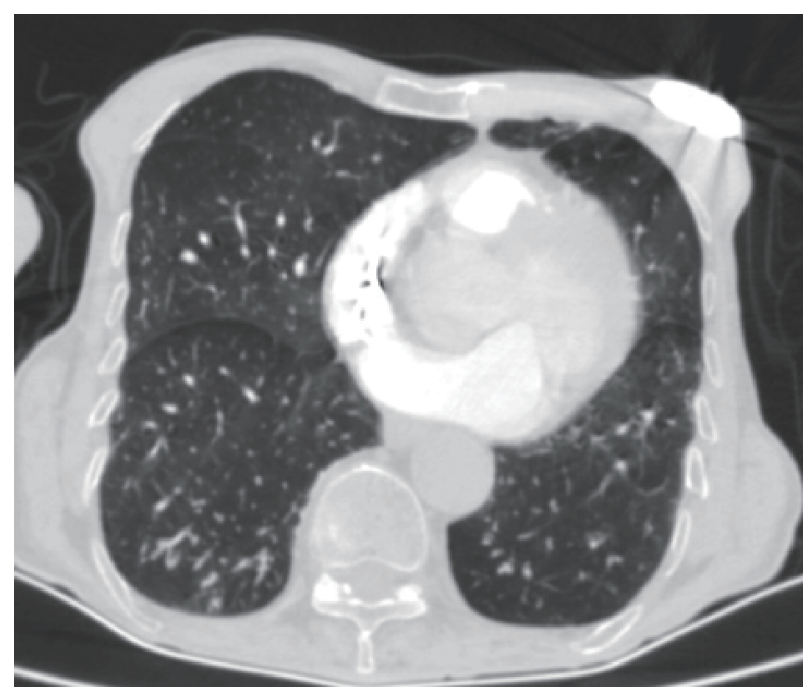

Figure 2. Three Weeks after beginning of treatment: complete regression. It appears that all visible involvement ground glass patchy areas have disappeared 3 weeks after beginning of treatment.

major basic protein are enzymes that cause endothelial damage secreted by eosinophils and increase fibrosis, thrombosis and infarction [7].

\section{Discussion}

Lung involvement can be seen in 40-60\% of cases in HES. The most common respiratory symptom is the chronic and persistent cough. Lung involvement results in sputum, wheezing, and shortness of breath, which increases the suspicion of bronchial hyperreactivity. As with our patient, patients may be mistaken for asthma or COPD. However, pulmonary function tests typically reveal that there is no obstructive pulmonary disease [8]. In these patients, BAL can be performed and the presence of a large number of eosinophils can be demonstrated. BAL sampling could not be done in our patient due to the risks in the pandemic situation.

When cardiac involvement occurs, the most common form is endomyocardial fibrosis. Progressive scarring, especially in the posterior mitral valve, mostly limits the movement of the valve. Although cardiac involvement is best diagnosed by endomyocardial biopsy, this procedure carries high morbidity and mortality risks. Instead, a noninvasive procedure, such as transesophageal echocardiography, is valuable for assessment and detection of possible thrombus formation. Chronic heart failure and severe arrhythmia are frequently found in the late stages of the HES, as in our case [7, 9, 10]. Neuropsychiatric symptoms are common in HES due to the involvement of the central nervous system [9]. It often presents with loss of consciousness, depressed mood, poor coordination, and peripheral neuropathy. There was no evidence of neuropsychiatric involvement in the hospital application and anamnesis in our patient.

Laboratory evaluation of primary eosinophilia should be initiated by screening factor interacting with PAPOLA and CPSF1 (FIP1L1)-PDGFR $\alpha$ gene fusion in peripheral blood. The presence of a deletion in $4 \mathrm{q} 12$ can be detected, leading to 
the formation of FIP1L1-PDGFR fusion [9].

Treatment should begin in HES, which includes organ damage by definition. The main treatment is corticosteroids; it is usually started at a dose of $1 \mathrm{mg} / \mathrm{kg}$, after 1 - 2 weeks of use at this dose, it is gradually reduced over a period of 2 - 3 months [3]. Imatinib is the first drug to be considered in the presence of translocations with other genes and changes in FIP1L1-PDGFR $\alpha$ or PDGFR $\beta$. Tyrosine kinase inhibitor therapy results in complete clinical and molecular remission in a high proportion of patients and complications associated with the disease, including transformation into leukemia, are prevented [3]. However, a small number of patients may require treatment changes and additional treatment.

\section{Conclusions}

HES can occur with nonspecific multiple cutaneous, immunological, pulmonary, cardiac, ocular, rheumatological and gastrointestinal manifestations. Physicians should definitely consider HES in the differential diagnosis of patients presenting with heart failure, pulmonary involvement and eosinophilia. As in our case, although other comorbidities, such as COPD, may prevent a correct diagnosis of HES with pulmonary involvement, early diagnosis usually provides the most appropriate treatment.

\section{Acknowledgments}

We remember all our colleagues who lost their lives due to COVID-19.

\section{Financial Disclosure}

No funding was received. None of the authors have disclosures relevant to this manuscript.

\section{Conflict of Interest}

None to declare.

\section{Informed Consent}

An informed consent was obtained from our patients to publish this study.

\section{Author Contributions}

All authors contributed to the editing of the manuscript. IS wrote the manuscript and created samples from computed tomography images.

\section{Data Availability}

The authors declare that data supporting the findings of this study are available within the article.

\section{References}

1. Shomali W, Gotlib J. World Health Organization-defined eosinophilic disorders: 2019 update on diagnosis, risk stratification, and management. Am J Hematol. 2019;94(10):1149-1167.

2. Tefferi A, Patnaik MM, Pardanani A. Eosinophilia: secondary, clonal and idiopathic. Br J Haematol. 2006;133(5):468492.

3. Tefferi A, Gotlib J, Pardanani A. Hypereosinophilic syndrome and clonal eosinophilia: point-of-care diagnostic algorithm and treatment update. Mayo Clin Proc. 2010;85(2):158-164.

4. Crane MM, Chang CM, Kobayashi MG, Weller PF. Incidence of myeloproliferative hypereosinophilic syndrome in the United States and an estimate of all hypereosinophilic syndrome incidence. J Allergy Clin Immunol. 2010;126(1):179-181.

5. Gotlib J, Cools J, Malone JM, 3rd, Schrier SL, Gilliland DG, Coutre SE. The FIP1L1-PDGFRalpha fusion tyrosine kinase in hypereosinophilic syndrome and chronic eosinophilic leukemia: implications for diagnosis, classification, and management. Blood. 2004;103(8):2879-2891.

6. Ogbogu PU, Bochner BS, Butterfield JH, Gleich GJ, Huss-Marp J, Kahn JE, Leiferman KM, et al. Hypereosinophilic syndrome: a multicenter, retrospective analysis of clinical characteristics and response to therapy. J Allergy Clin Immunol. 2009;124(6):1319-1325 e1313.

7. De Cock C, Lemaitre J, Deuvaert FE. Loeffler endomyocarditis: a clinical presentation as right ventricular tumor. J Heart Valve Dis. 1998;7(6):668-671.

8. Klion AD, Robyn J, Akin C, Noel P, Brown M, Law M, Metcalfe DD, et al. Molecular remission and reversal of myelofibrosis in response to imatinib mesylate treatment in patients with the myeloproliferative variant of hypereosinophilic syndrome. Blood. 2004;103(2):473-478.

9. Mor A, Segev A, Hershkovits R, Lew S, Mekori YA. Hypereosinophilic syndrome presenting as acute myocardial infarction. Allergy. 2000;55(9):899-900.

10. Cools J, DeAngelo DJ, Gotlib J, Stover EH, Legare RD, Cortes J, Kutok J, et al. A tyrosine kinase created by fusion of the PDGFRA and FIP1L1 genes as a therapeutic target of imatinib in idiopathic hypereosinophilic syndrome. N Engl J Med. 2003;348(13):1201-1214. 